\title{
EL BACINETE EN LA ETIOPATOGENIA DE LAS FISTULAS UROGENITALES
}

\author{
Doctores B. Neme, C. Paula Martins, L. Mathias y J. Gallucei *
}

\section{CONSIDERACIONES}

En los países de gran extensión territorial y de bajo índice demográfico, el parto representa aún el principal factor etiopatogénico de las fístulas uro-genitales.

Considerando informes, exclusivamente a partir de 1941 y concernientes a publicaciones brasileras, indias, argentinas y peruanas [Lucas Machado (14), Krishnan (12), Reyes (20), Neme y Gallucci (19), Azevedo (2), So uza Rudge (21), Clovis Salgado (23), Burgos (4), Couri (7), Martiniano Fernandes y Sa Leitao (9), Guixá y Otturi (11), Lana (13), Salaber y Guixá (22) y Thompson (26)], verificamos que el factor obstétrico incidió en un mínimo en $28,9 \%$, en un máximo en $100 \%$ y, en promedio, más o menos en $90 \%$ de los casos de fístulas uro-genitales.

Dos mecanismos explican la etiopatogenia de las lesiones tocológicas: la compresión o necrosis y el traumatismo quirúrgico.

La mayoría de los autores admite que es la necrosis isquémica, consecuente a los partos prolongados, la causa más frecuente de solución de continuidad del tracto uro-genital. La afirmación de Fritsch (1899) (10) de que en la etiopatogenia de las fístulas tocogenéticas la tendencia intervencionista tendría mayor responsabilidad que la actitud expectante, encuentra oposición en los informes de Mahfouz (15) (1930), Souza Rudge (21) (1941), Neme y Gallucci (19) (1953), Salgado (23) (1960) y Machado (14) (1961), según los cuales el factor necrosis por compresión estuvo presente, respectivamente, en $95 \%$, $88,1 \%, 49 \%, 62,1 \%, 83,6 \%$ y $90,5 \%$ de los casos observados.

La anomalía pélvica, dificultando el tránsito fetal, favorece la compresión de los tejidos génito-urinarios del canal del parto $y$, más por su duración que por la intensidad (Mahfouz (16), 1938), contribuye para su isquemia y mortificación.

Sabiéndose desde largo tiempo que la anomalía de la pelvis es una ocurrencia frecuente entre las portadoras de fístula tocogenética, por su efecto estancante de la progresión fetal, es extrañable que

* Departamento de Obstetricia y Ginecología de la Facultad de Medicina de la Universidad de Sao Paulo (Servicio del Profesor José Medina). 
no se haya hecho ningún estudio radiológico en relación con las pelvis de estas pacientes. De la misma manera que sobre el aspecto clínico, eran muy vagas las referencias de la literatura sobre las características dimensionales y morfológicas de estas pelvis.

Para Zangemeister, citado por Stoeckel (25) (1948), tendría fundamental importancia, para la producción de necrosis de compresión, la forma y el tamaño del estrecho superior. El caso sería más grave en el bacinete achatado que en el general y regularmente estrecho, porque en aquél la compresión se haría por la sínfisis, mientras que en el último, la misma compresión se distribuiría a lo largo del segmento relativamente largo del estrecho superior.

Igual opinión fue sustentada por Bumm (3) (1923) y, en general, repetida en los demás tratados y publicaciones referentes al tema.

Finalmente, Moir (18) (1961) notó que el achatamiento de la pelvis raramente estuvo presente, mientras que la existencia de arco púbico agudo fue siempre la regla.

Por estas razones nos pareció útil el estudio que ahora emprendemos.

\section{MATERIAL}

Estudiamos 21 pacientes portadoras de fístula uro-genital, internadas en el Departamento de Obstetricia y Ginecología de la Universidad de Sao Paulo (Servicio del profesor Medina), en el período de 1951-58. En todas, la lesión del tracto urinario se relacionó con el parto. Considerando la duración del trabajo del parto y la época de aparición de la incontinencia urinaria, admitimos a la necrosis de compresión como causa determinante de la lesión fistulosa.

\section{METODO DE ESTUDIO}

Las pacientes fueron sometidas a radipelvimetría, por la técnica de ThomsWilson, que consta de dos proyecciones: 1a supero-inferior del estrecho superior, en posición semi-sentada, y $2^{a}$, lateral, de toda la pelvis en posición de pies.

Fueron determinadas las dimensiones de los estrechos y el tipo de bacinete. En el estrecho superior fueron medidos los diámetros conjugado verdadero y el transverso máximo. En el estrecho medio el bi-espinoso, el anteroposterior y el sagital posterior. En relación con el estrecho inferior, consideramos la medida de la arcada púbica y la distancia sínfiso-biparietal.

El tipo de bacinete fue determinado según la clasificación de Caldwell y cols. (5).

\section{RESULTADOS}

En la tabla I presentamos los datos relacionados al examen radiopelvimétrico. Para la evaluación de la capacidad pélvica consideramos, como válidos, los criterios de Eastman (8), de WeinbergScadron (29), y de Allen (1).

En relación con el estrecho superior, Eastman (8) admite ser de $9,5 \mathrm{~cm}$ la medida mínima normal para el conjugado verdadero; para el estrecho medio, el mismo autor considera presente la distocia cuando el bi-espinoso mide $9,0 \mathrm{~cm}$ o menos. 
TABLA I

\section{RESULTADOS DEL EXAMEN RADIOPELVIMETRICO}

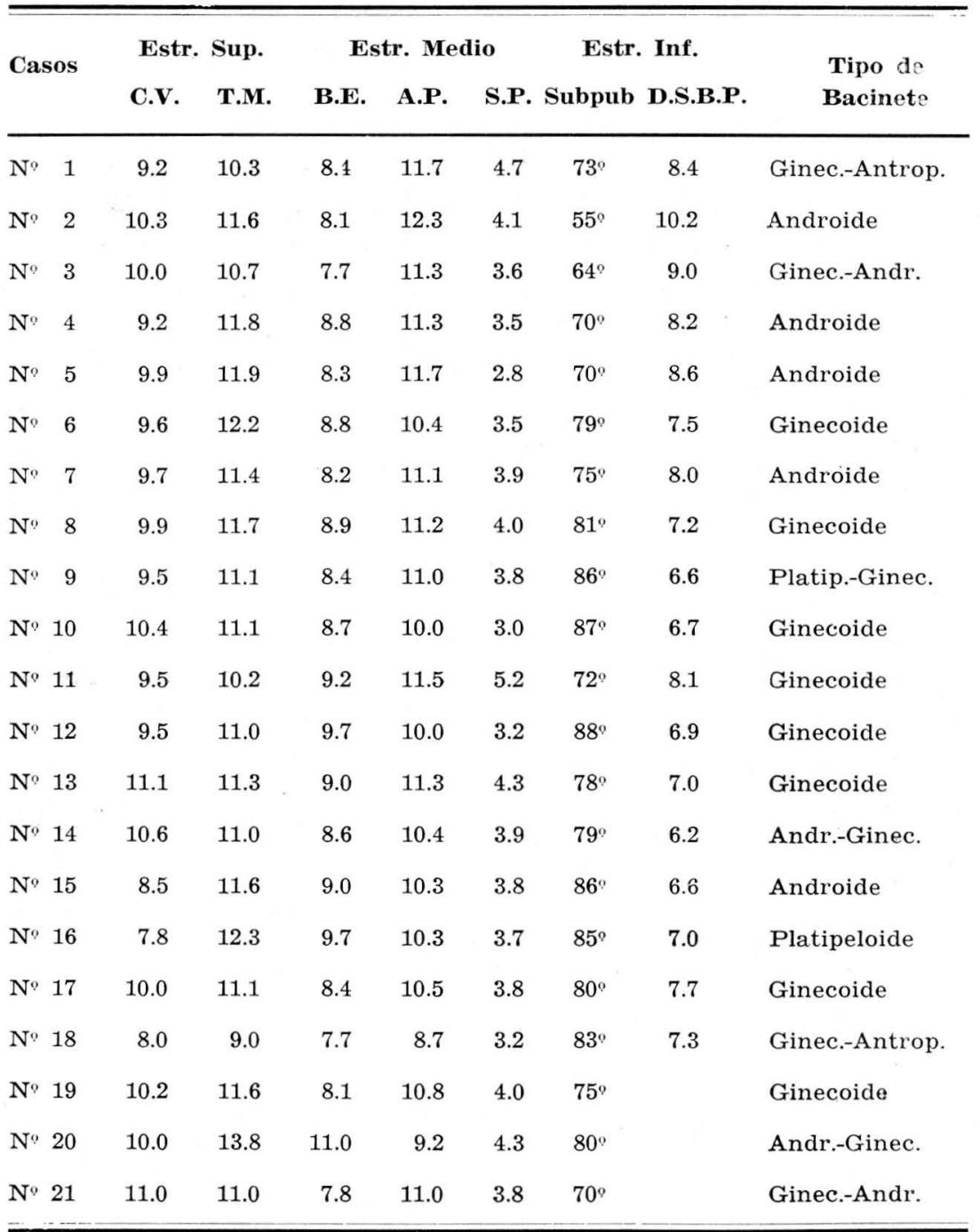

Admitiendo la idea de que entre los diámetros perpendiculares de la pelvis puede ocurrir una compensación recíproca, Weinberg-Scadron (29) establecieron el criterio que se fundamenta en la suma de sus valores. Así, en relación con el estrecho superior, $20 \mathrm{~cm}$ sería el límite mínimo normal; para el estrecho medio la suma del bi-espinoso y sagital posterior no sobrepasaría de 13 a $13,5 \mathrm{~cm}$. 
TABLA II

INCIDENCIA DE CONSTRICCION PELVICA

Estrecho-Superior Estrecho Medio $\begin{gathered}\text { Estrecho } \\ \text { Inferior }\end{gathered}$
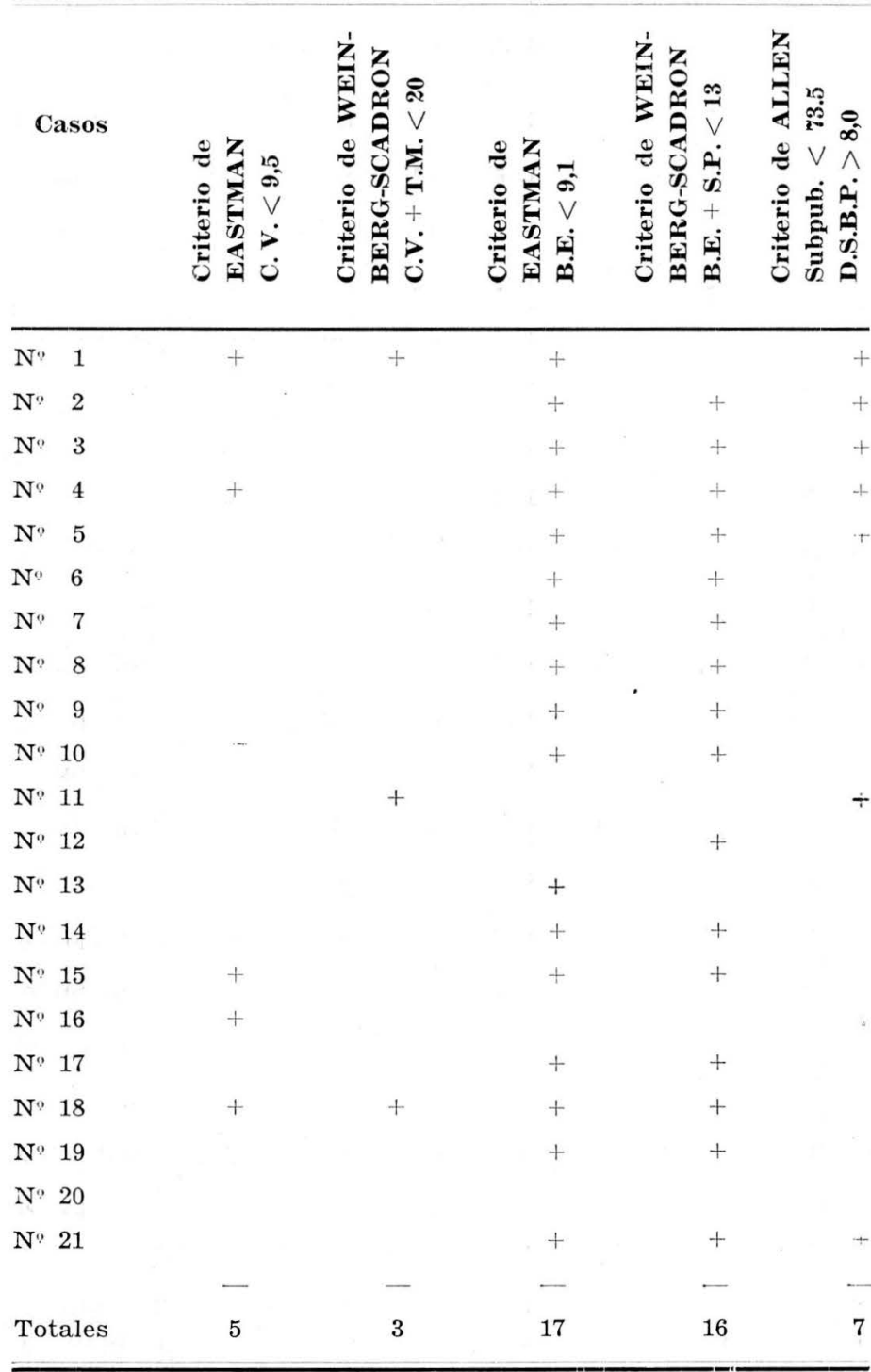

Finalmente, de acuerdo con Allen superior a $8 \mathrm{~cm}$, son indicativos de dis(1), el ángulo subpúbico inferior a tocia del estrecho inferior. $73,5^{\circ}$ y la distancia sínfiso-biparietal 
En la tabla II verificamos la incidencia de constricción pélvica en nuestros casos, aplicando los criterios ya mencionados.

El examen de las tablas I y II demuestra ser la distocia del estrecho medio, prácticamente, la regla en los casos de fístulas uro-genitales tocogenéticas que estudiamos.

En atención a los criterios a que hemos hecho referencia, verificamos que mientras las distocias del estrecho superior e inferior estuvieron presentes, respectivamente, en $23,8 \%$ y $33,3 \%$, aquella del estrecho medio incidió en un $85,7 \%$ de nuestros casos.

\section{DISCUSION}

El examen de la tabla II demuestra que en los casos de fístulas uro-genitales tocogenéticas, por necrosis, la anomalía pélvica constituye factor indiscutible. Por otro lado, el análisis de las dimensiones correspondientes a los tres estrechos del bacinete permite verificar el papel preponderante de la distocia del estrecho medio en la determinación de estas lesiones.

Atendiendo las observaciones de $\mathrm{Sa}$ vage (24), quien sostiene que la distocia del estrecho superior es más frecuente que la del medio y, comúnmente, se presenta aislada, queda claro que la elevada incidencia de la estrechez en el plano medio representa, en nuestros casos, la condición determinante en la detención del tránsito fetal.

Por otra parte, dos factores, relacionados con la anomalía del estrecho medio, guardan importancia en la etiopatogenia de las fístulas tocogenéticas por necrosis. El primero relaciona la mayor incidencia de las occipitoposteriores en casos de biespinoso disminuído (presente en $85,7 \%$ de nuestros casos); el segundo deriva de la aparente insinuación de la presentación, que suele ocurrir cuando existe la distocia aislada del estrecho medio. El primer factor, dificultando la rotación interna, prolonga la evolución del parto y, el segundo predispone a los tocólogos con poca experiencia a seguir una conducta expectante por vía baja. La compresión prolongada del tracto uro-genital favorece la necrosis isquémica. La frecuente localización de la fístula, a nivel del cuello vesical, según los estudios de Malpas y cols. (17), explícase por el desplazamiento de la vejiga, que, mediante la progresión cefálica, se dirige hacia arriba exponiendo su base a la compresión ósea, cuando la presentación se-halla enclavada en el bacinete. Con relación al tipo de bacinete, algunos hechos llaman la atención: Como se observa en la tabla I, el tipo platipeloide se encuentra apenas en un caso. Este es un hecho que nos sorprende, teniendo en cuenta las ideas de Zangmeister, ya mencionadas. Por otro lado, se nota que el tipo antropoide no está representado, lo que confirma la opinión de Thoms (28) (1947), según la cual sería éste el tipo de bacinete más eutócico. Se verifica, igualmente, que el tipo androide puro o asociado, considerado altamente distócico, estuvo presente en 9 de nuestros 21 casos, proporción que, en nuestro medio, admitimos estar por encima del índice promedio. Finalmente, el análisis de los valores relacionados con el ángulo sub-púbico, confirma las sospechas de Moir, para quien, en los 
casos de fístula uro-genital obstétrica, por necrosis, la presencia de arco púbico agudo (menos de $90^{\circ}$ ) fue la regla.

En nuestros casos este hecho se verificó en $100,0 \%$.

\section{CONCLUSIONES}

El examen radiopelvimétrico de 21 portadoras de fístula uro-genital tocogenética, por necrosis, nos permitió las siguientes conclusiones:

1a En cinco casos $(23,8 \%)$ se halló reducción de los diámetros correspondientes al estrecho superior.

$2^{a}$ En 18 casos $(85,7 \%)$ se constató la presencia de angostamiento de! estrecho medio.

$3^{\text {a }}$ En 7 casos $(33,3 \%)$ el estrecho inferior apareció con reducción de su capacidad. 4ạ El predominio de constricción (en frecuencia e intensidad) al nivel del estrecho medio, sobre los estrechos superior e inferior, muestra que a aquella distocia debe caber la mayor responsabilidad en la etiopatogenia de las fístuias obstétricas, por necrosis.

5a El diagnóstico más preciso y precoz de la distocia del estrecho medio permite la profilaxis de cierto número de fístulas tocogenéticas, por necrosis y quirúrgicas. En el primer caso no se permitirá la progresión indebida del parto $y$, en el segundo, el tocólogo se abstendrá de distender exageradamente los tejidos maternos, previamente lesionados por la isquemia compresiva.

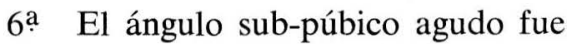
la regla en los bacinetes de los casos estudiados de fístula uro-genital tocogenética, por necrosis.

\section{$\begin{array}{llllllllllll}\text { B } & \text { I } & \text { B } & \text { L } & \text { I } & \mathbf{O} & \mathbf{G} & \mathbf{R} & \mathbf{A} & \mathbf{F} & \mathbf{I} & \mathbf{A}\end{array}$}

1. ALlen, E. P.: Brit. J. Radiology 20: 164; 1947.

2. AZEVEDO, G. V.: An. Bras. Ginec. 17: 1; 1944.

3. BUMm, E.: Trattado Completo de Ostetricia. Soc. Edit. Libraria, Milán; 1923.

4. BURgos, J. F.: Tratamento das Fístulas Vésico-Genitais. Tese Fac. Med. Univ. Bahía, 1953.

5. Caldwell, W. E., MOLOY, H. C. and D'ESOPO, A.: Am. J. Obst. \& Gynec., 40: 558; 1940.

6. ChassarD, M., et LAP.NE, M.: J. Radiol. et D'electrologie 7: 113; 1923.

7. COURI, A. A.: An. Bras. Ginec. 34: $25 ; 1952$.

8. EAStMAN, N.: Williams Obstetries. Appleton-Century-Crofts, New York, 1956.

9. Fernandes M. y SA Leitao, A.: An. Bras. Ginec. 30: 31; 1950.

10. FRITSCH, H.: Deutsch. Med. Wschr. 1: 199; 1899.

11. Guixa, H. L., y otturi, J. E.: Sem. Méd. 104: 27; 1954.

12. KRIShNAN, R. G.: J. Obst. Gynec. Brit. Emp. 56: 22; 1949.

13. LANA, F. V.: Rev. Ginec. d’Obst. 48: 733; 1954. 
14. MACHADO, L. M.: A propósito de Fístulas uro-genitais. Información personal, 1961.

15. MAHFOUZ, N.: J. Obst. Gynaec. Brit. Emp. 37: 566; 1930.

16. Mahfouz, N.: J. Obst. Gynaee. Brit. Emp. 45: 405; 1938.

17. MAlpas, P., JefFCoATE, T. N. A., and LISTER, U. N.: J. Obst. Gynaec. Brit. Emp. 5: $49 ; 1949$.

18. MOIR, J. C.: The Vesico-Vaginal Fistula, Bailliére Tindall \& Cox, London, 1961.

13. Neme, B. e Gallucci, J.: Rev. Hosp. N. S. Aparecida 6: 45; 1953.

20. Reyes, N. V.: Rev. Med. Peruana 21: 183; 1949.

21. RUDGE, W. S.: Contribuicäo para o tratamento das Fistulas Uro-genitais pela plastica do desdobramento. Tese Fac. Med. Univ. S. Paulo, 1941.

22. Salaber, J. A. y Guixa, H. L.: Prensa Méd. Argeni. 41: 247; 1954.

23. SALGADO, C.: Memoria presentada a Acad. Nac. Medicina, 1960.

24. Savage, J. E.: Am. J. Obst. Gynec. 61: 809: 1951.

2b. StOECKEL, W.: Tratado de Ginecologia, Edic. Morata, 2a. ed., Madrid, 1948.

26. Thompson, D. J.: J. Obst. Gynaec. Brit. Emp. 52 : 271; 1945.

27. THOMS, H.: Am. J. Obst. \& Gynec. 42: 947; 1941.

28. THOMS, H.: Am. J. Obst. \& Gynec. 54: 62; 1947.

29. WeinberG, A., and SCADRON, S. J.: Am. J. Obsi. \& Gynec. 52: 255; 1946. 